\title{
An Intensified Vibratory Milling Process for Enhancing the Breakage Kinetics during the Preparation of Drug Nanosuspensions
}

\author{
Meng Li, ${ }^{1}$ Lu Zhang, ${ }^{1}$ Rajesh N. Davé, ${ }^{1}$ and Ecevit Bilgili ${ }^{1,2}$
}

Received 9 May 2015; accepted 7 July 2015; published online 17 July 2015

\begin{abstract}
As a drug-sparing approach in early development, vibratory milling has been used for the preparation of nanosuspensions of poorly water-soluble drugs. The aim of this study was to intensify this process through a systematic increase in vibration intensity and bead loading with the optimal bead size for faster production. Griseofulvin, a poorly water-soluble drug, was wet-milled using yttrium-stabilized zirconia beads with sizes ranging from 50 to $1500 \mu \mathrm{m}$ at low power density $(0.87 \mathrm{~W} / \mathrm{g})$. Then, this process was intensified with the optimal bead size by sequentially increasing vibration intensity and bead loading. Additional experiments with several bead sizes were performed at high power density $(16 \mathrm{~W} / \mathrm{g})$, and the results were compared to those from wet stirred media milling. Laser diffraction, scanning electron microscopy, X-ray diffraction, differential scanning calorimetry, and dissolution tests were used for characterization. Results for the low power density indicated $800 \mu \mathrm{m}$ as the optimal bead size which led to a median size of $545 \mathrm{~nm}$ with more than $10 \%$ of the drug particles greater than $1.8 \mu \mathrm{m}$ albeit the fastest breakage. An increase in either vibration intensity or bead loading resulted in faster breakage. The most intensified process led to $90 \%$ of the particles being smaller than $300 \mathrm{~nm}$. At the high power intensity, $400 \mu \mathrm{m}$ beads were optimal, which enhanced griseofulvin dissolution significantly and signified the importance of bead size in view of the power density. Only the optimally intensified vibratory milling led to a comparable nanosuspension to that prepared by the stirred media milling.
\end{abstract}

KEY WORDS: bioavailability enhancement; drug nanoparticles; LabRAM; process intensification; vibratory milling.

\section{INTRODUCTION}

The number of poorly water-soluble drug candidates coming out of drug discovery has increased significantly $(1,2)$. The bioavailability of poorly water-soluble drugs is limited by their solubility and dissolution rate (3), which poses great challenge to pharmaceutical formulators in their development. One way for increasing the bioavailability of such drugs is to increase the surface area via formation of nanoparticles. Size reduction of drug crystals increases the specific surface area, which can improve the dissolution rate of drugs $(4,5)$, according to the Noyes-Whitney equation (6). The drug nanoparticles in suspension form are mostly incorporated into standard solid dosage forms such as capsules, tablets, sachets (7-10), and recently into strip films (11) upon drying. Furthermore, drug nanoparticles are also amenable to systemic delivery via parenteral or inhalation administration $(12,13)$.

\section{Meng Li and Lu Zhang contributed equally to this work.}

\footnotetext{
${ }^{1}$ Otto H. York Department of Chemical, Biological and Pharmaceutical Engineering, New Jersey Institute of Technology, Newark, New Jersey, USA.

${ }^{2}$ To whom correspondence should be addressed. (e-mail: bilgece@njit.edu)
}

Drug nanoparticles can be produced by "bottom-up" or "top-down" methods, or their combinations $(13,14)$. The bottom-up approach refers to methods that create small drug particles from drug molecules dissolved in a solvent through nucleation mechanism (15), such as liquid anti-solvent precipitation (16), precipitation using supercritical fluid (17), and evaporative precipitation (18). However, complexity of the process control and potential risk of residual organic solvents as well as low drug loading and physical stability issues have discouraged the development of commercial products (19). In the top-down approach, two size reduction methods have been commonly used: wet stirred media milling (20) and high-pressure homogenization (21). Both of these methods require high energy consumption and long cycle time especially at the manufacturing scale. Another disadvantage of wet media milling is the risk of contamination due to media wear (22) and phase transition of drug induced by high mechanical stresses (23). Despite these disadvantages, wet stirred media milling has been preferred over other top-down methods in the pharmaceutical industry as it is continuous, scalable, solvent-free, and environmentally benign (24-27). Moreover, nanosuspensions prepared via wet media milling have the distinct advantages of high drug loading, low excipient side effects, and can be generally formulated for most drug candidates (28). Several drug nanoparticle-based products, making use of wet stirred media milling, have been 
approved by the US Food and Drug Administration as oral products $(15,29)$.

In drug discovery and early development phase, the availability of drug substance is limited, thus entailing the use of drug-sparing methods for identification of a suitable nanosuspension formulation and/or evaluation of multiple drug candidates. Leung et al. (30) reported the resonant acoustic mixing of drug suspensions with milling media (beads), which allowed for streamlined identification of an optimal drug nanosuspension formulation. The acoustic mixing has been previously used for liquid mixing $(31,32)$, powder mixing $(33,34)$, and dry coating of drugs and excipients (35-38). In this process, low-frequency, high-intensity vibratory energy is applied to the entire mixing vessel to homogeneously mix the components while the vessel oscillates at the resonance frequency (39-41). Leung et al. (30) has successfully adapted the resonant-vibratory mixing to the production of drug nanosuspensions with the use of beads in the mixing vessel, and this process will be referred to as the vibratory milling throughout this paper. Interestingly, the impact of process parameters on the breakage kinetics and milled particle size distribution (PSD) has not been investigated at all. Moreover, Leung et al. (30) reports wet-milling of drugs under a very narrow set of vibratory milling conditions. Process parameters such as bead size, vibration intensity, and bead loading can significantly affect the breakage rate and milling time required for a desired fineness. In addition, design and optimization of any wet media milling process entails a good understanding of the breakage kinetics and its controlling process parameters (24). While process parameters of the wet stirred media milling, the most commonly used process for preparing drug nanosuspensions, have been widely studied $(24,42,43)$ and the process has been intensified via a novel model-guided approach (44), no such investigation for the vibratory milling exists.

The aim of this study was to intensify the vibratory milling process through a systematic investigation of the impact of vibration intensity, bead loading, and bead size for faster production of drug nanosuspensions. Griseofulvin (GF) particles were wet-milled in the presence of two stabilizers, hydroxypropyl cellulose (HPC) and sodium dodecyl sulfate (SDS), to prepare stable GF suspensions under various processing conditions. Laser diffraction (LD), scanning electron microscopy (SEM), powder X-ray diffraction (PXRD), differential scanning calorimetry (DSC), and dissolution tests were used to characterize the particles and their performance. First, several milling experiments were performed to determine the optimal bead size at low power density $P_{\mathrm{w}}$. Then, the process was intensified with the optimal bead size and operated at a much higher $P_{\mathrm{w}}$ by increasing vibration intensity and bead loading step-wise with the objective of increasing the breakage rate. After examining the temporal evolution of the particle size upon intensification, additional experiments were performed with various bead sizes to explore if and how the optimal bead size depends on the power density. Finally, a comparative assessment of the performance of vibratory milling $v s$. wet stirred media milling has been carried out in view of the power density, resultant breakage kinetics, and final drug particle size attained. This study provides a systematic process intensification approach as well as guidance for bead size selection and suggests process/design improvement strategies for fast preparation of drug nanosuspensions via vibratory milling.

\section{MATERIALS AND METHODS}

\section{Materials}

Griseofulvin (GF, BP/EP grade) was purchased from Letco Medical (Decatur, AL, USA). GF is a Biopharmaceutics Classification System (BCS) Class II drug with an aqueous solubility of $7.7 \mu \mathrm{g} / \mathrm{ml}$ (20). It was selected here as a model BCS Class II drug because it was used in various wet stirred media milling studies $(8,9,45)$, which helps to select the stabilizer formulation rationally and allows for comparative assessment. Hydroxypropyl cellulose (HPC, SL grade), which is commonly used as a neutral polymeric stabilizer, was donated by Nisso America Inc. (New York, NY, USA). Sodium dodecyl sulfate (SDS), which is an anionic surfactant, was purchased from Sigma-Aldrich (Milwaukee, WI, USA). Its critical micelle concentration (CMC) in water is $8.2 \mathrm{mM}(0.24 \% w / w)$ at ambient temperature. Zirmil Y grade wear-resistant yttrium-stabilized zirconia beads (YSZ) with nominal sizes of 50, 200, 400, 800, and $1500 \mu \mathrm{m}$ were purchased from Saint Gobain ZirPro (Mountainside, NJ, USA) and used as the milling media. Throughout the paper, the beads were labeled with their nominal sizes, while their actual median sizes are 54, 214, 396, and $802 \mu \mathrm{m}$, respectively, which were measured in dry dispersion mode via a laser diffraction (LD) particle size analyzer (Helos/Rodos, Sympatec, NJ, USA). Due to the limitation of Helos/Rodos, actual size of $1500 \mu \mathrm{m}$ beads was measured using wet dispersion mode via LS 13320 (Coulter Beckman, Brea, CA, USA) with Fraunhofer method. Its actual median size was found to be $1458 \mu \mathrm{m}$. Deionized water was used in all milling and particle sizing experiments.

\section{Preparation of Suspensions via Vibratory Milling}

Table I presents the process parameters as well as amounts of drug suspension and slurry (drug suspension with beads) used in the milling experiments. In all suspensions, GF, HPC, and SDS concentrations were kept at 10,2.5, and $0.05 \%$, respectively, based on the excellent physical stability of the GF nanosuspensions prepared with HPC-SDS combination via wet media milling (45). Here, all percentages in formulations are $w / w$ with respect to deionized water. A $25-\mathrm{ml}$ glass vial with cap was chosen as the mixing/milling vessel. First, an aqueous HPC-SDS solution was prepared using the LabRAM resonant-vibratory mixer (Resodyn Acoustic Mixers, Inc., Butte, MT, USA) by adding HPC and SDS into deionized water, followed by mixing at vibration intensity $I$ of $30 \%$ for 20 min. After defoaming at room temperature, GF and YTZ beads were added in sequence to the stabilizer solution and mixed manually by shaking for 2 min so as to prepare GF presuspensions. The total volume of the slurry was maintained at about $90 \%$ of the vial volume by adjusting the amount of suspension (see Table I for the suspension amount) due to the different bead size and bead loading $\phi$, which refers to the percentage of the vial volume occupied by the beads. Small amount (1.2-1.8 g) of GF was used in each milling experiment.

Drug pre-suspensions prepared were then milled in the LabRAM vibratory mixer for $96 \mathrm{~min}$ at the conditions presented in Table I. Frequency was set at $61 \mathrm{~Hz}$, and acceleration $a$ of the oscillatory motion of the vessel, which was 
Table I. Process Parameters, Suspension Mass, and Slurry Mass Varied in the Vibratory Milling Experiments

\begin{tabular}{|c|c|c|c|c|c|c|}
\hline Run & Nominal bead size, $D_{\mathrm{b}}$ & Intensity, $I$ & Acceleration, $a$ & Bead loading $^{b}, \phi$ & Suspension mass ${ }^{c}$ & Slurry mass \\
\hline No. ${ }^{a}$ & $(\mu \mathrm{m})$ & $(\%)$ & $\left(\mathrm{m} / \mathrm{s}^{2}\right)$ & $(\%)$ & $(\mathrm{g})$ & $(\mathrm{g})$ \\
\hline 1 & 50 & 40 & $50 g$ & 50 & 17.2 & 60.5 \\
\hline 2 & 200 & 40 & $50 g$ & 50 & 16.6 & 63.2 \\
\hline 3 & 400 & 40 & $50 g$ & 50 & 16.8 & 63.7 \\
\hline 4 & 800 & 40 & $50 g$ & 50 & 16.6 & 62.5 \\
\hline 5 & 1500 & 40 & $50 g$ & 50 & 16.5 & 63.1 \\
\hline 6 & 800 & 60 & $75 g$ & 50 & 16.6 & 62.5 \\
\hline 7 & 800 & 75 & $90 g$ & 50 & 16.6 & 62.5 \\
\hline 8 & 800 & 90 & $100 \mathrm{~g}$ & 50 & 16.6 & 62.5 \\
\hline 9 & 800 & 90 & $100 g$ & 30 & 20.1 & 47.9 \\
\hline 10 & 800 & 90 & $100 \mathrm{~g}$ & 70 & 13.1 & 78.5 \\
\hline 11 & 50 & 90 & $100 g$ & 70 & 13.9 & 74.6 \\
\hline 12 & 200 & 90 & $100 \mathrm{~g}$ & 70 & 13.1 & 78.9 \\
\hline 13 & 400 & 90 & $100 \mathrm{~g}$ & 70 & 12.9 & 78.3 \\
\hline
\end{tabular}

${ }^{a}$ Fixed parameters: GF loading of $10 \%$, HPC/SDS concentration of $2.5 \% / 0.05 \%$ (weight percent with respect to deionized water), milling time of 96 min

${ }^{b}$ Percent bulk volume of the beads with respect to the vial volume

${ }^{c}$ Adjusted for some milling experiments due to changes in bead size-loading to ensure that the slurry occupies $90 \%$ of the vial volume in all experiments

recorded from the control panel of the equipment, increased proportionally to the set values of $I$. Essentially, $I$ modulates $a$ and vibration energy at fixed frequency. The power consumption $P$ and power density $P_{\mathrm{w}}$, i.e., power consumption per unit mass of drug, were calculated using the Excel-based code provided by Resodyn Acoustic Mixers, Inc., which takes into account the measured values of vessel mass, slurry mass, $I$, and $a$. In the first part of this study, the impact of bead size $D_{\mathrm{b}}$ at low $P_{\mathrm{w}}(\sim 0.87 \mathrm{~W} / \mathrm{g})$ was studied through Runs $1-5$, keeping $I$ and $\phi$ constant, which were taken from the study by Leung et al. (30). Then, following the recently proposed novel process intensification approach for wet stirred media milling (44), $I$ and $\phi$ were sequentially increased (Runs $6-10$ with the exception of Run 9) using the optimal bead size identified from Runs 1-5. The intensification aims to enhance breakage kinetics and increase product fineness for a given milling time. Additional milling experiments (Runs 11-13) were performed with several bead sizes at the high $P_{\mathrm{w}}(\sim 16 \mathrm{~W} / \mathrm{g})$ to explore the dependence of optimal bead size on $P_{\mathrm{w}}$. In exploratory experiments without external cooling, significant temperature rise occurred due to viscous heat dissipation during the milling; e.g., in Run 5 , the slurry temperature rose to $58^{\circ} \mathrm{C}$ within 32 min of milling. In view of this, to maintain the slurry temperature below $35^{\circ} \mathrm{C}$ throughout 96 min milling, an intermittent cooling strategy (similar to Afolabi et al. (24)) was followed for all experiments: at $8 \mathrm{~min}$ intervals, the glass vial was dipped into a refrigerated bath (NESLAB RTE 10, Thermo Scientific, Newington, NH, USA). Following each cooling, the milling was continued until the GF particles were subjected to milling action for a total duration of $96 \mathrm{~min}$ in all milling experiments.

Suspension samples were taken from the supernatant in the vial after various milling times and used for particle size analysis. The relatively "fine" suspensions with median particle sizes $D_{50}$ below $0.5 \mu \mathrm{m}$ were refrigerated at $8^{\circ} \mathrm{C}$ for 7 days. Drug particle size statistics obtained immediately after milling and after 7-day storage were compared to assess the shortterm physical stability of the suspensions. In general, it was assumed that the milled suspensions were intended to be dried shortly after milling (see e.g., refs. $(8,9)$ ), justifying the 7-day stability.

\section{Preparation of Suspensions via Wet Stirred Media Milling}

Wet-milling was performed in a Microcer stirred media mill (Netzsch Fine Particle Size Technology, LLC, Exton, PA, USA) to make a performance comparison with vibratory milling using $400 \mu \mathrm{m}$ YTZ beads. About 226 g GF pre-suspension with identical formulation/ composition to that used in vibratory milling was prepared and then milled at the conditions selected based on our previous work (45), i.e., stirrer speed of $11.7 \mathrm{~m} / \mathrm{s}$, bead loading of $196 \mathrm{~g}$, and suspension flow rate of $126 \mathrm{ml} / \mathrm{min}$. The amounts of the drug suspension and slurry inside the 80-ml milling chamber were calculated to be 53.7 and $250 \mathrm{~g}$, respectively. Both the milling chamber and the holding tank are equipped with a chiller unit (model number M1-.25A-11HFX, Advantage Engineering, Greenwood, IN, USA) that kept the suspension temperature in the holding tank below $35^{\circ} \mathrm{C}$. Details of the equipment and milling procedure can be found elsewhere $(24,45)$. The suspensions at several milling time points were collected from the outlet of milling chamber for particle size analysis. The total energy consumption $E$ was directly recorded from the mill control panel. Using this information, the average power density per unit mass of drug $P_{\mathrm{w}}$ was calculated as follows:

$P_{w}=E /\left(t_{\mathrm{T}} m_{\text {Drug }}\right)$

where $t_{\mathrm{T}}$ is the total milling time and $m_{\text {Drug }}$ is the drug mass inside the milling chamber. 


\section{Characterization}

Particle size distributions (PSDs) of the milled suspensions and 7-day stored suspensions were measured using laser diffraction (LS 13 320, Coulter Beckman, Brea, CA, USA). A polarized intensity differential scattering (PIDS) obscuration water optical model was employed. The PIDS was maintained between 40 and $50 \%$ while the obscuration was maintained below $8 \%$. PSD was computed by the equipment's software using the Mie scattering theory. Refractive index values are 1.65 and 1.33, respectively, for GF and the measurement medium (deionized water). Prior to the size measurement, $\sim 0.2 \mathrm{ml}$ samples of the suspensions were diluted with $0.4 \mathrm{ml}$ solution of HPC-SDS. SEM imaging was used to examine the morphology of the GF particles before and after milling. About $1 \mathrm{ml}$ aliquot of the finest drug suspension prepared via the vibratory milling, i.e., Run 13 suspension, was diluted into $30 \mathrm{ml}$ deionized water, vortex-mixed for $30 \mathrm{~s}$, mounted on a silicon chip (Ted Pella, Inc., Redding, CA, USA), placed on top of carbon specimen holders, and dried in a desiccator. The samples were then sputter coated with carbon and examined under a LEO 1530 SVMP (Carl Zeiss, Inc., Peabody, MA, USA).

The crystallinity of the as-received drug, unmilled physical mixture of GF-HPC-SDS, and overnight dried Run 13 suspension was analyzed using PXRD (PANalytical, Westborough, MA, USA), provided with $\mathrm{Cu} \mathrm{K}_{\alpha}$ radiation ( $\lambda=1.5406 \AA)$. The samples were scanned for $2 \theta$ ranging from 5 to $40^{\circ}$ at a scan rate of $0.165 \mathrm{~s}^{-1}$. The same samples were also subjected to differential scanning calorimetry (DSC) and USP II dissolution tests; except that Run 13 suspension was tested without overnight drying for the dissolution test. A MettlerToledo polymer analyzer (Model: PolyDSC, Columbus, $\mathrm{OH}$, USA) was used for DSC. The samples were heated at a rate of $10^{\circ} \mathrm{C} / \mathrm{min}$ with a temperature range of $25-250^{\circ} \mathrm{C}$ under nitrogen flow. The peak melting point temperature $T_{\mathrm{m}}$ and fusion enthalpy $\Delta H_{\mathrm{m}}$ were determined using the equipment's software. The dissolution test was conducted via a Distek 2100C dissolution tester (North Brunswick, NJ, USA) according to the USP II paddle method. The dissolution medium was $1000 \mathrm{ml}$ deionized water that was maintained at $37^{\circ} \mathrm{C}$, and a paddle speed of $50 \mathrm{rpm}$ was used. The samples were weighed equivalent to a GF dose of $8.9 \mathrm{mg}$. They were poured into the dissolution medium, and $4 \mathrm{ml}$ samples were taken out manually at 1, 2, 5, 10, 20, 30, and $60 \mathrm{~min}$. These aliquots were filtered through a $0.1 \mu \mathrm{m}$ PVDF membrane-type syringe filter, and the amount of GF dissolved was determined by UV spectroscopy (Agilent, Santa Clara, CA, USA) at a wavelength of $296 \mathrm{~nm}$ using a previously established calibration curve. The average of three replicates from each sample was reported along with a standard deviation.

\section{RESULTS AND DISCUSSION}

\section{Impact of Bead Size at Low Power Density}

Proper selection of bead size $D_{\mathrm{b}}$ can have significant impact on the breakage kinetics and final milled particle size during vibratory milling. GF particles were wet-milled in the vibratory mixer (LabRAM) using beads with different nominal sizes: 50, 200, 400, 800, and $1500 \mu \mathrm{m}$ at the low power density $P_{\mathrm{w}}$ of $0.87 \pm 0.02 \mathrm{~W} / \mathrm{g}$ (refer to Table I, Runs 1-5). Figure 1 presents the temporal evolution of the characteristic particle sizes $D_{50}$ and $D_{90}$ during the milling with different bead sizes. Since it is well-established that the combined use of HPC-SDS imparts excellent stability to nanosuspensions of GF $(24,45)$ and multiple BCS Class II drugs (46), a shallower slope of the $D_{50} v s$. time curves or greater $D_{50}$ at select milling times indicates slower particle breakage and lower breakage rate. As seen from Fig. 1, the GF particle size decreased monotonically during the whole milling except for $50 \mu \mathrm{m}$ beads whose use led to negligible extent of breakage. After the fast initial breakage within the first 8 min which can be attributed to the fast breakage of relatively weak large crystals, the overall breakage rate tended to decrease with prolonged milling. Table II presents the particle sizes and their standard deviation (SD) for 96 min milled suspensions. At the low power density $P_{\mathrm{w}}$, sub-micron GF particles in terms of $D_{50}$ were only produced when $800 \mu \mathrm{m}$ beads were used, whereas small beads (50 and $200 \mu \mathrm{m}$ ) were ineffective for breaking the GF particles. Except $1500 \mu \mathrm{m}$ beads, larger beads led to the formation of finer drug particles and faster breakage. The data presented in Fig. 1 and Table II overall suggest that similar to the wet stirred media milling process $(47,48)$, an optimal bead size exists for the vibratory milling process and that $800 \mu \mathrm{m}$ beads were optimal at the low power density.

The origin of the optimal bead size warrants some serious consideration. It is known that smaller beads have a higher number concentration than larger beads for a given mass loading of the beads. Li et al. (44) proposed a microhydrodynamic explanation for the two major counteracting effects of colliding milling beads in a wellmixed vessel such as a stirred media mill: on one hand, the fluctuating motion of the smaller beads was less vigorous, which led to lower maximum bead contact pressure upon bead-bead collisions in the mill. On the other hand, the frequency of bead-bead collisions and drug particle compression events increased dramatically with a decrease in bead size due to the higher number concentration associated with smaller beads. Considering that the vibratory mixing of the beads also led to significant bead-bead collisions and capture/ compression of the drug particles leading to breakage, the aforementioned counteracting effects are also valid, at least qualitatively, for the vibratory milling, which is the origin of the optimal bead size. This size is expected to depend upon which one of the two counteracting effects above is more pronounced and how they relate to the mechanical properties of the specific drug. In view of these counteracting effects, results from Fig. 1 can be interpreted as follows: small beads (50 and $200 \mu \mathrm{m}$ ) did not lead to energetic collisions and sufficiently high mechanical stresses (stress intensity), whereas $1500 \mu \mathrm{m}$ beads did not lead to sufficient number of bead-bead collisions (stressing number); apparently, $800 \mu \mathrm{m}$ beads led to optimal stress intensity-number, leading to the fastest kinetics at the low power density regime. It should be mentioned that in the low power density experiments (Runs 1-5), the bulk volume of the beads was kept at $50 \%$ with respect to vial volume, which corresponded to a small variation of the bead mass loading, i.e., $45.9 \pm 1.5 \mathrm{~g}$. Therefore, the slight variation of the bulk volume of the various bead sizes used is expected to 


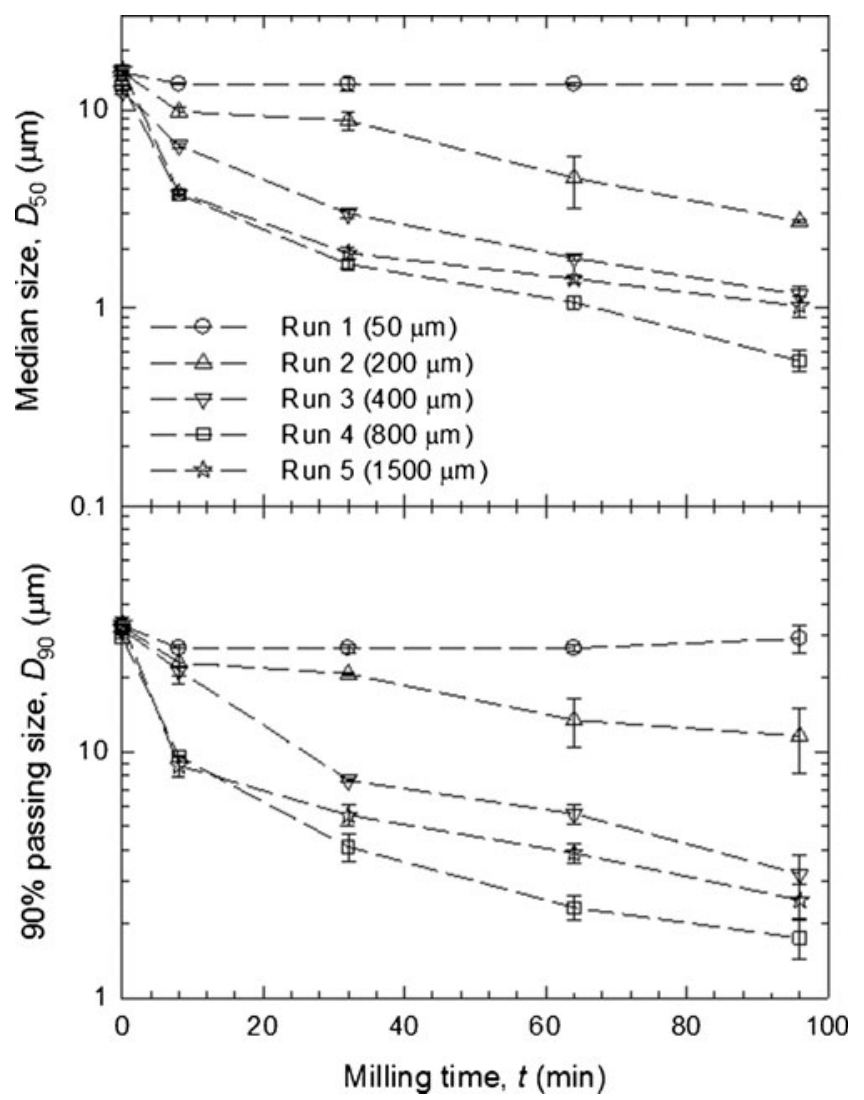

Fig. 1. Effect of bead size $D_{\mathrm{b}}$ on the temporal evolution of GF particle sizes at low power density $P_{\mathrm{w}}$ (Runs $1-5$ ): vibration intensity $I=40 \%$ and bead loading $\phi=50 \%$

play a negligible role as compared with the 30 -fold change in bead size in the respective experiments.

\section{Impact of Process Intensification}

Despite being optimal at the low power density, $800 \mu \mathrm{m}$ beads resulted in a relatively coarse nanosuspension with a median size of $545 \mathrm{~nm}$ and more than $10 \%$ of the particles greater than $1.8 \mu \mathrm{m}$, which necessitates enhancement of the breakage kinetics. The baseline process conditions used in this study leading to the relatively low power density were taken from Leung et al. (30). To enhance breakage kinetics and thus produce drug nanoparticles faster, the impact of vibration intensity $I$ and (volumetric) bead loading $\phi$ were investigated following a process intensification strategy similar to that

Table II. Particle Sizes and Their Standard Deviation $S D$ after Milling, Power Consumption $P$, and Power Density $P_{\mathrm{w}}$ for Runs $1-14$

\begin{tabular}{|c|c|c|c|c|}
\hline Run & $D_{50} \pm S D$ & $D_{90} \pm S D$ & $P$ & $P_{\mathrm{w}}$ \\
\hline No. & $(\mu \mathrm{m})$ & $(\mu \mathrm{m})$ & $(\mathrm{W})$ & $(\mathrm{W} / \mathrm{g})$ \\
\hline 1 & $13.38 \pm 0.714$ & $26.86 \pm 3.755$ & 1.3 & 0.85 \\
\hline 2 & $2.745 \pm 0.054$ & $11.56 \pm 3.370$ & 1.3 & 0.88 \\
\hline 3 & $1.168 \pm 0.108$ & $3.185 \pm 0.640$ & 1.3 & 0.87 \\
\hline 4 & $0.545 \pm 0.066$ & $1.751 \pm 0.320$ & 1.3 & 0.88 \\
\hline 5 & $1.026 \pm 0.124$ & $2.497 \pm 0.400$ & 1.3 & 0.89 \\
\hline 6 & $0.446 \pm 0.013$ & $1.516 \pm 0.072$ & 2.9 & 2.0 \\
\hline 7 & $0.395 \pm 0.005$ & $1.317 \pm 0.054$ & 7.6 & 5.2 \\
\hline 8 & $0.365 \pm 0.006$ & $1.213 \pm 0.238$ & 18 & 12 \\
\hline 9 & $0.450 \pm 0.005$ & $1.503 \pm 0.066$ & 18 & 10 \\
\hline 10 & $0.312 \pm 0.031$ & $0.560 \pm 0.047$ & 18 & 16 \\
\hline 11 & $1.438 \pm 0.030$ & $5.524 \pm 0.159$ & 18 & 15 \\
\hline 12 & $0.301 \pm 0.001$ & $1.257 \pm 0.080$ & 18 & 15 \\
\hline 13 & $0.185 \pm 0.010$ & $0.296 \pm 0.033$ & 18 & 16 \\
\hline $14^{\mathrm{a}}$ & $0.158 \pm 0.001$ & $0.240 \pm 0.001$ & 25 & 5.2 \\
\hline
\end{tabular}

${ }^{a}$ Prepared via wet stirred media milling 
developed for wet stirred media milling (44). The wet media milling process was intensified through sequential increase in $I$ and $\phi$, which resulted in a higher power density $P_{\mathrm{w}}$ (see Table II).

Figure 2a shows the effects of $I$ on temporal evolution of GF particles during 96 min milling in Runs 4 and 6-8. An increase in $I$ led to faster particle breakage and consequently smaller final particles. However, it should be noticed that $90 \%$ cumulative passing size $\left(D_{90}\right)$ of final drug particle size was still above $1 \mu \mathrm{m}$ even at the highest $I$. Further intensification was achieved by exploring the impact of $\phi$ in Runs 9 and 10 at the highest vibration intensity used, i.e., 90\% (Fig. 2b), and it was found that an increase in $\phi$ also enhanced the breakage kinetics, further contributing to process intensification. Among Runs 1-10, only Run 10 with the highest $I$ and $\phi$ (most intensified process) led to $D_{90}$ below $1 \mu \mathrm{m}$, i.e., $0.560 \mu \mathrm{m}$, within $96 \mathrm{~min}$ of milling. The $D_{50}$ and $D_{90}$ values for the repeat of this most intensified process were 0.314 and $0.557 \mu \mathrm{m}$, respectively. The deviations of the repeat particle size values were less than $1 \%$, signifying the reproducibility of the vibratory milling, which accords well with the findings of Leung et al. (30). Short-term physical stability was studied only for the milled suspensions whose $D_{50}$ were below $0.5 \mu \mathrm{m}$, i.e., Runs 6-10, 12, and 13 suspensions, by storing respective suspension samples for 7 days at $8^{\circ} \mathrm{C}$. Figure 3 shows that the particle size did not change significantly after 7-day storage.

Let us attempt to explain the significant impact of $I$ and $\phi$. It should be noted that during the process intensification via an increase in $I$ with $800 \mu \mathrm{m}$ beads, $P_{\mathrm{w}}$ increased
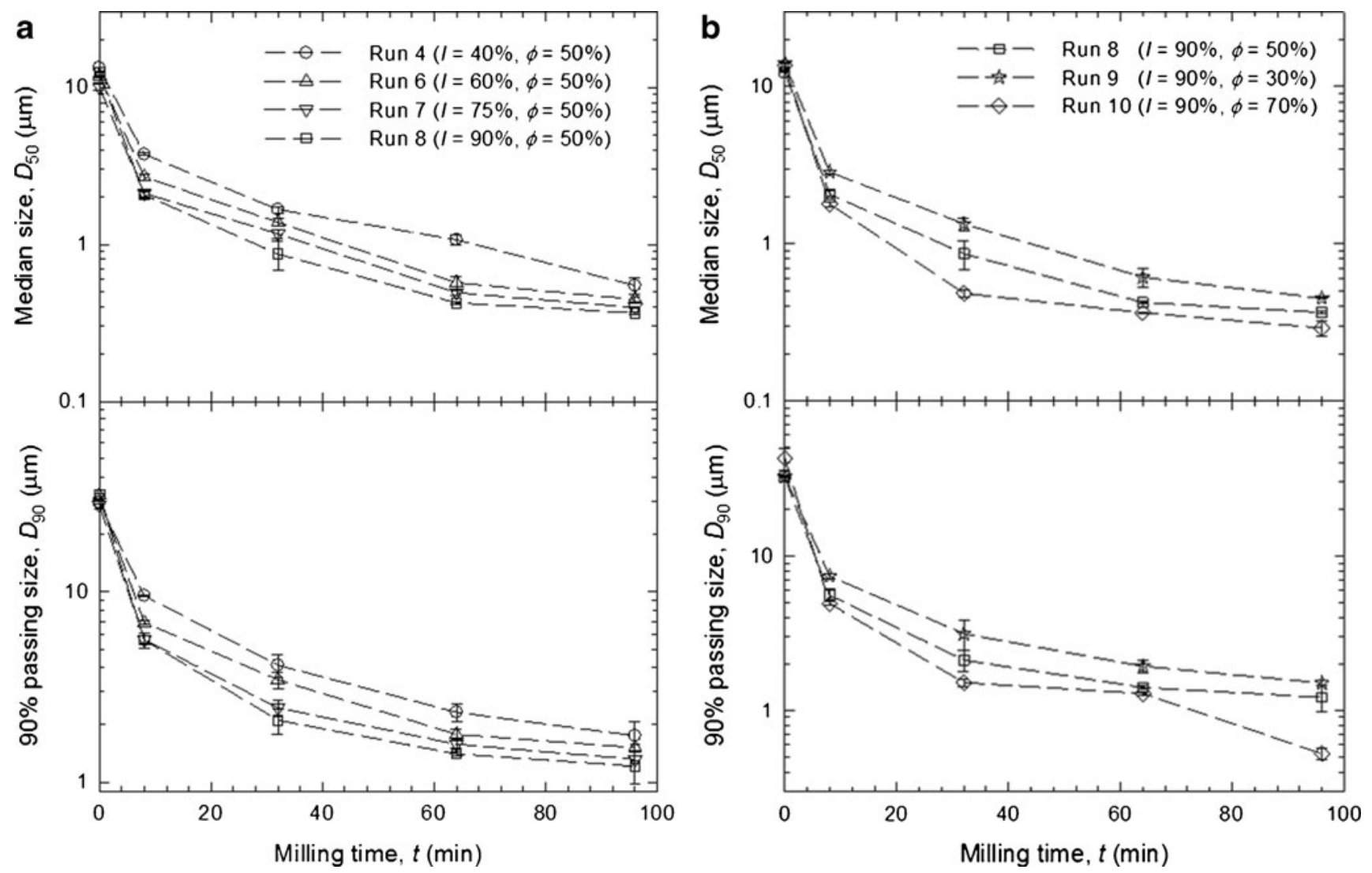

Fig. 2. Effects of a vibration intensity I (Runs 4, 6-8) and b bead loading $\phi$ (Runs 8-10) on the temporal evolution of GF particle sizes with the use of $800 \mu \mathrm{m}$ YSZ beads significantly (Table II), which is expected to increase the stress intensity-number of colliding beads in general. Some useful insight may be gained by extending the comprehensive microhydrodynamic analysis of the impact of rotor speed and bead loading in wet stirred media milling (24) to the vibratory milling with the beads. It is likely that at higher $I$ (analogy to higher rotor speed in a wet stirred media mill), average bead oscillation velocity and maximum bead contact pressure were higher, which led to the enhanced breakage kinetics and finer particles. Interestingly, Afolabi et al. (24) reported counteracting effects for the impact of $\phi$ for a media mill: at higher $\phi$, the stress intensity decreased while the number of stressing events dramatically increased (24), noting the former effect is unfavorable for faster breakage, whereas the latter effect is favorable. A secondary, yet another positive effect of higher $\phi$ is that upon an increase in $\phi$, the mass of suspension and drug both decreased and $P_{\mathrm{w}}$ increased consequently (Tables I and II), which could explain the reduced milling time required for a desired fineness (24).

\section{Dependence of Optimal Bead Size on Power Density}

In this section, the effects of bead size $D_{\mathrm{b}}$ on the temporal evolution of GF particles during 96 min milling were investigated at the high $P_{\mathrm{w}}(\sim 16 \mathrm{~W} / \mathrm{g})$ in Runs $10-13$ and illustrated in Fig. 4 with final milled sized reported in Table II. We again find that an optimal bead size exists, i.e., $400 \mu \mathrm{m}$ (Run 13), which was smaller than the optimal bead size at the low $P_{\mathrm{w}}$ $(0.87 \mathrm{~W} / \mathrm{g})$, i.e., $800 \mu \mathrm{m}$ (Run 4), suggesting a dependence on $P_{\mathrm{w}}$. Repeat of the milling with the optimal bead size (Runs 13) 


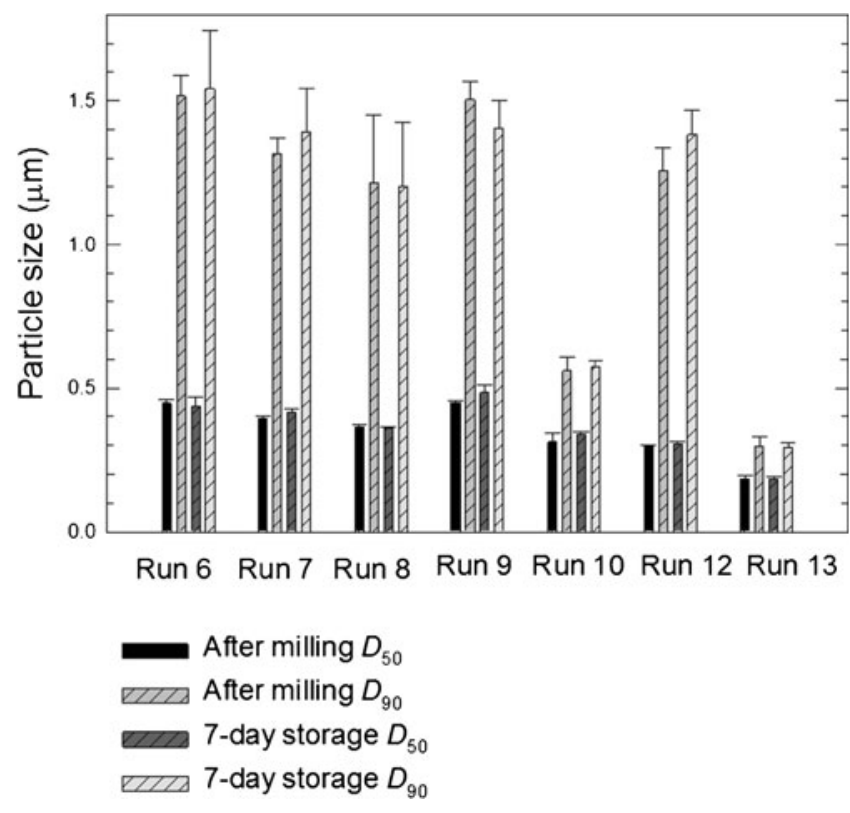

Fig. 3. Drug particle sizes after milling and after 7-day storage for Runs 6-10, 12, and 13

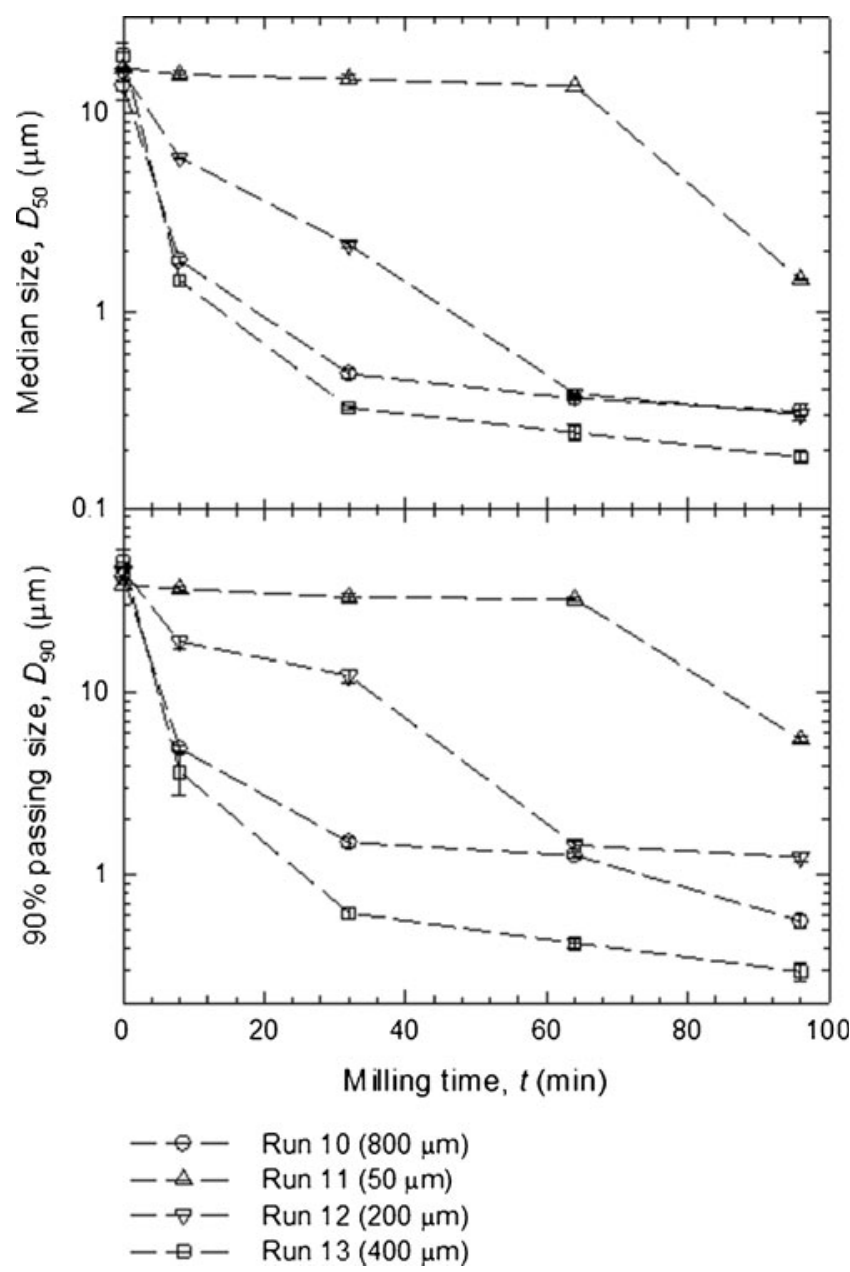

Fig. 4. Effect of bead size $D_{\mathrm{b}}$ on the temporal evolution of GF particle sizes at high power density $P_{\mathrm{w}}$ (Runs $10-13$ ): vibration intensity $I=90 \%$ and bead loading $\phi=70 \%$ 

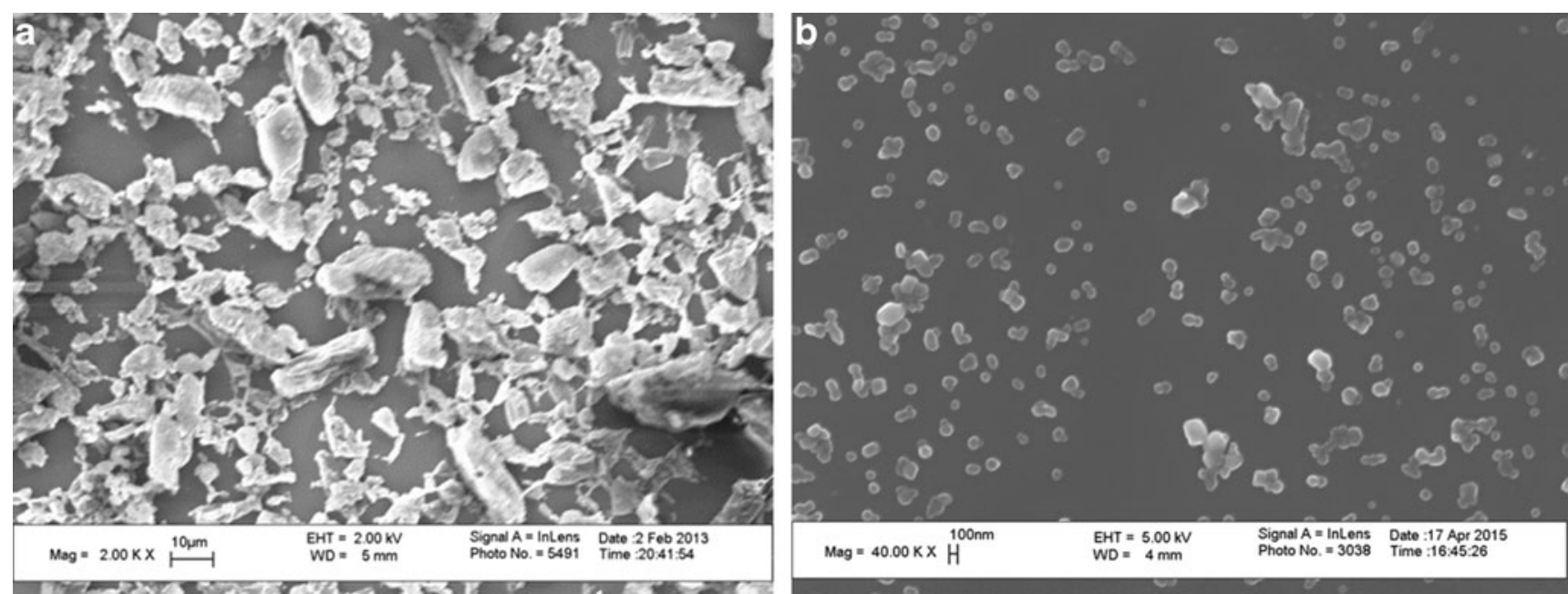

Fig. 5. SEM images of GF particles: a before milling (marker size $10 \mu \mathrm{m}, \times 2 \mathrm{k}$ magnification) and b after milling in Run 13 (marker size: $100 \mathrm{~nm}$, $\times 40 \mathrm{k}$ magnification). Run 13 refers to milling with $400 \mu \mathrm{m}$ YSZ beads in the vibratory mill operating at high power density $P_{\mathrm{w}}(I=90 \%, \phi=70 \%)$

had $D_{50}$ and $D_{90}$ of 0.183 and $0.289 \mu \mathrm{m}$, respectively, with less than $\sim 2 \%$ deviation from the original milling experiment,

a

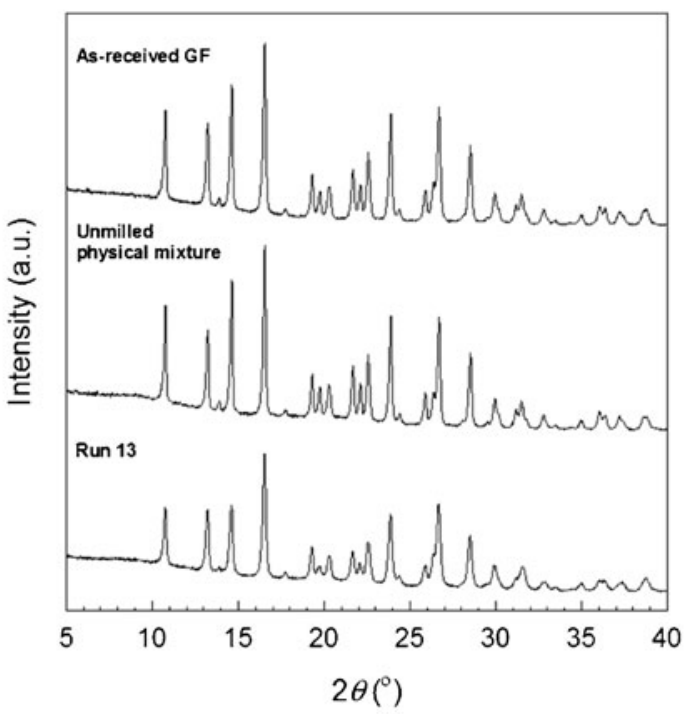

C

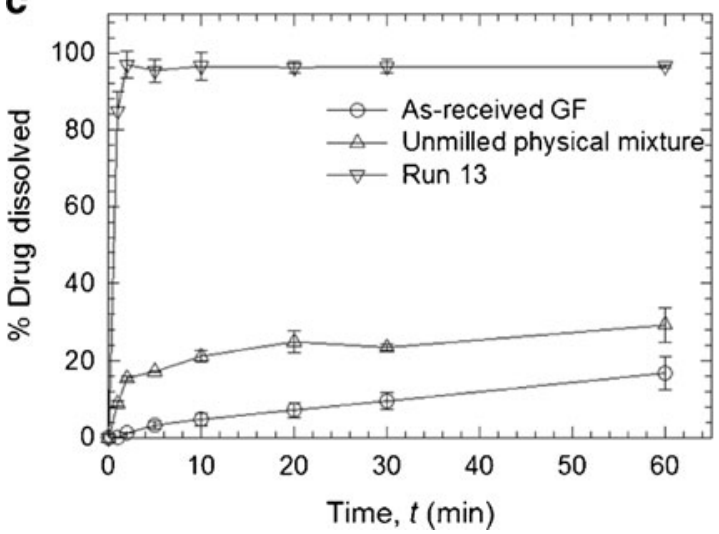

suggesting the reproducibility again. A comparison of Figs. 1 and 4 for the same bead size reveals that the breakage rate

b

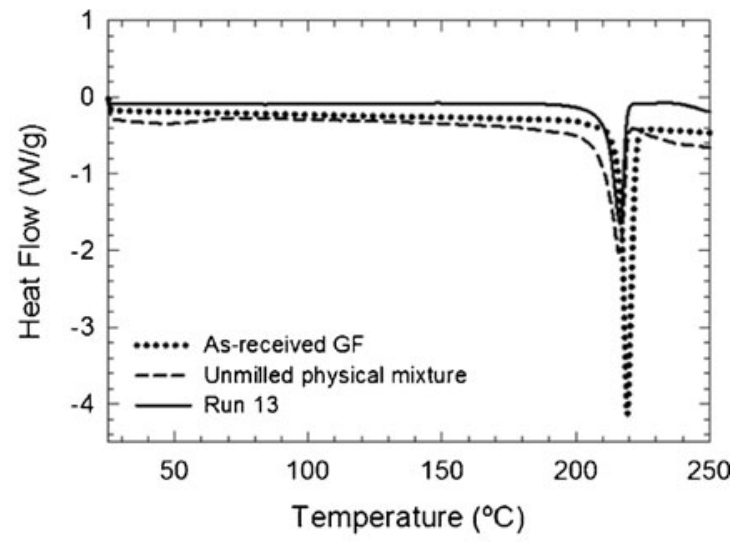

Fig. 6. a PXRD diffractograms, b DSC thermograms, and $\mathbf{c}$ GF dissolution profiles for as-received GF, unmilled physical mixture, and dried milled suspensions prepared with $400 \mu \mathrm{m}$ YSZ beads. Run 13 refers to 96 min milling in the vibratory mill operating at high power density $P_{\mathrm{w}}(I=90 \%, \phi=70 \%)$ 
was much faster at the higher $P_{\mathrm{w}}$ (more intensified). A significant change to $D_{50}$ and $D_{90}$ for Runs 10-13 suspensions over 7-day storage was not observed (see Fig. 3).

In general, a higher $P_{\mathrm{w}}(15.5 \pm 0.6 \mathrm{~W} / \mathrm{g})$ was applied to the system in Runs 10-13 (Table II) than that in Runs $1-5$ (0.87 $\pm 0.02 \mathrm{~W} / \mathrm{g}$ ), which led to faster breakage for a given bead size. This could be explained as follows: it is likely that a higher $P_{\mathrm{w}}$ could increase average bead oscillation velocity, maximum bead contact pressure, and number of stressing events, which all favor drug particle capture and breakage. The shift of optimal bead size from 800 to $400 \mu \mathrm{m}$ at higher $P_{\mathrm{w}}$ seems to be elusive at first. In the section Impact of Bead Size at Low Power Density, the impact of $D_{\mathrm{b}}$ at low $P_{\mathrm{w}}$ was discussed at length and two counteracting effects of $D_{\mathrm{b}}$ were pointed out: smaller beads have higher number concentration leading to a higher bead oscillation velocity and number of drug particle compression events, but lower maximum contact pressure (lower stress intensity or less forceful collisions). Depending on which of these two counteracting effects is more pronounced, the optimal $D_{\mathrm{b}}$ can shift (44). A higher $P_{\mathrm{w}}$ could have increased the contact pressure and oscillation velocity of $400 \mu \mathrm{m}$ beads to such a level that, coupled with their higher number concentration as compared with $800 \mu \mathrm{m}$ beads, rendered them optimal.

\section{Further Characterization of the GF Particles}

The vibratory milling of the as-received GF particles (Fig. 5a) led to formation of more rounded particles (Fig. 5b). The primary GF particles in Fig. 5b appear to be in the approximate size range of $0.10-0.40 \mu \mathrm{m}$, which confirms the production of nanoparticles after 96 min vibratory milling in Run 13. Particle sizes observed from SEM qualitatively and measured from LD quantitatively were close, which suggests that severe aggregation of GF particles did not take place and the use of HPC-SDS can stabilize GF nanoparticles, which confirms the proper selection of the stabilizer system per Afolabi et al. (24).

Another potential concern with wet media milling is that the intensified vibratory milling process may lead to significant changes in the crystalline state of drugs. Figure 6 a presents the

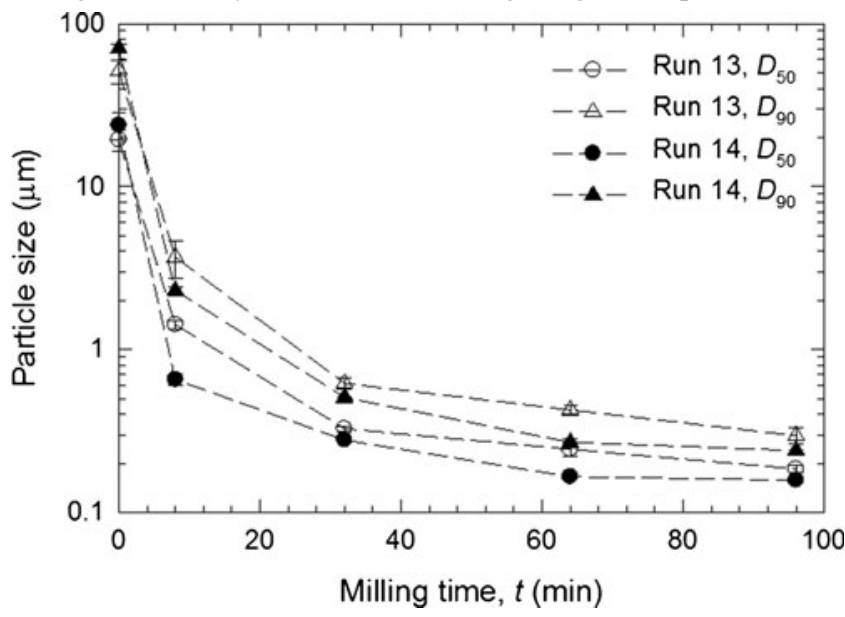

Fig. 7. Temporal evolution of GF particle sizes with $400 \mu \mathrm{m}$ YSZ beads in Run $13(I=90 \%, \phi=70 \%)$ using the vibratory mill and Run 14 (stirrer speed $=11.7 \mathrm{~m} / \mathrm{s}$, bead mass $=196 \mathrm{~g}$, flow rate $=126 \mathrm{ml} / \mathrm{min}$ ) using the wet stirred media mill
PXRD diffractograms of the as-received GF, unmilled physical mixture of GF with HPC-SDS, and 96 min milled GF suspension (Run 13) after overnight drying. Only Run 13 suspension was tested in PXRD-DSC among all vibratory milled suspensions because Run 13 is the most aggressive run causing the greatest damage to the particles with the fastest breakage (refer to Fig. 4). As compared to the diffractograms of both the as-received GF and the physical mixture, the peak positions remained the same with slightly lower peak intensity for Run 13 , which could be attributed to defect formation and accumulation during milling (27). DSC thermograms (Fig. 6b) of the same samples exhibited a notable endothermic event associated with the melting of the crystalline GF. Most importantly, the thermogram of the Run 13 sample was very similar to that of the physical mixture both having $T_{\mathrm{m}}$ of $216^{\circ} \mathrm{C}$, but the $\Delta H_{\mathrm{m}}$ of the former was slightly smaller $(66.4 \mathrm{~J} / \mathrm{g}$ vs. $75.6 \mathrm{~J} / \mathrm{g})$, most probably due to the aforementioned defect formation-accumulation during the milling and the presence of nanocrystals. Comparative analysis of these thermograms to that of the as-received GF with $T_{\mathrm{m}}=220^{\circ} \mathrm{C}$ and $\Delta H_{\mathrm{m}}=91.2 \mathrm{~J} / \mathrm{g}$ suggests that the reduced $\Delta H_{\mathrm{m}}$ and $T_{\mathrm{m}}$ upon milling could be mostly attributed to the dilution and encapsulation of the drug particles by the amorphous polymer (HPC). This finding accords very well with previous work (27) on the wet stirred media milling of GF, which showed identical DSC thermograms for the milled GF without stabilizers and the as-received GF. Overall, both the PXRD and DSC results here suggest that although vibratory media milling might have caused some defect formation in GF crystals, the crystalline nature of GF was largely preserved. This finding is not surprising because comprehensive characterization of the milled GF particles via DSC, XRD, and Raman spectroscopy demonstrated that even apparently more aggressive wet stirred media milling process did not cause notable change to the crystallinity $(27,44)$. Moreover, similar observations were made for the crystallinity of other wet media milled, poorly water-soluble drugs such as fenofibrate (49-51) and indomethacin (44).

Figure $6 \mathrm{c}$ illustrates the remarkably fast dissolution, i.e., complete dissolution within $2 \mathrm{~min}$, in water for the vibratory milled GF (Run 13) in comparison to the slow dissolution of the as-received GF and GF in the physical mixture. Although the physical mixture shows faster GF dissolution than the asreceived GF due to enhanced wettability imparted by the stabilizers (HPC-SDS), only $\sim 30 \%$ of GF dissolved after $60 \mathrm{~min}$. The remarkable enhancement for Run 13 originated from the 64-fold increase in the external specific surface area $A_{\text {ext }}$ of the GF particles from $0.36 \mathrm{~m}^{2} / \mathrm{g}$ (as-received GF) to $23.2 \mathrm{~m}^{2} / \mathrm{g}$ (Run 13), in accordance with the celebrated NoyesWhitney equation (6). Here, we assumed sphericity of the drug particles, which is only valid for an approximate analysis, and calculated $A_{\text {ext }}$ using $A_{\text {ext }}=6 /\left(\rho_{\mathrm{p}} D_{32}\right)$, where $\rho_{\mathrm{p}}$ is the true density of GF $\left(1.45 \mathrm{~g} / \mathrm{cm}^{3}\right)$ and $D_{32}$ is the Sauter mean diameter that was obtained from the respective laser diffraction measurement.

\section{A Comparison to Wet Stirred Media Milling}

GF particles having the same formulation (10\% GF, $2.5 \%$ HPC, and $0.05 \%$ SDS) were wet-milled for $96 \mathrm{~min}$ in the LabRAM vibratory mixer (Run 13) and a Netzsch wet stirred 
media mill (Run 14) both using $400 \mu \mathrm{m}$ YTZ beads for a proper comparative assessment. Run 13 was chosen as the best vibratory milling run among Runs 1-13 as it resulted in the fastest breakage and smallest GF particles. It is the most intensified vibratory milling run with the optimal bead size at high $P_{\mathrm{w}}$. The temporal evolution of GF particles during $96 \mathrm{~min}$ is shown in Fig. 7, and final particle sizes after 96 min are listed in Table II. Wet stirred media milling (Run 14) was faster and more efficient than the vibratory milling (Run 13) considering that the former processed a much larger suspension batch $(226 \mathrm{~g})$ than the latter $(12.9 \mathrm{~g})$. Also note that Run 14 had a lower $P_{\mathrm{w}}$ (Table II) and lower volumetric bead concentration $c$, which is the ratio of the true bead volume to the total vial/ chamber volume, than Run 13: 0.408 vs. 0.442. Despite the favorable kinetics expected from higher $c$ and/or $P_{\mathrm{w}}$ for a smaller suspension mass (batch size) in the vibratory milling process (Run 13), Fig. 7 portrays a contrary picture. This discrepancy may be explained by the insight gained from recent microhydrodynamic studies $(24,44,52)$; while $P_{\mathrm{w}}$ is an important parameter that controls many microhydrodynamic parameters in a given geometry/scale of a wet media mill, the actual specific energy per unit mass used for deforming the drug particles is a very small fraction of $P_{\mathrm{w}}$. Moreover, how the total mechanical energy is spent in a mill is a complex function of the mill geometry, rheology of the suspension, and the boundary conditions imposed by a moving wall or stirrer. The results presented in this section suggest that the wet stirred media mill uses $P_{\mathrm{w}}$ more effectively. Obviously, a microhydrodynamic analysis of the vibratory mill is needed either through computational fluid dynamic or simpler microhydrodynamic models, which is beyond the scope of this study.

While this section indicates the superiority of the wet stirred media milling, it is of utmost importance to mention that the vibratory milling is still advantageous as a drugsparing approach in early drug development (30), e.g., Run 13 used about $6 \%$ of the drug used in Run 14 . On the other hand, the faster breakage kinetics and higher efficiency associated with wet stirred media milling renders it more attractive for preparation of clinical supplies and manufacturing at the large scale. Among all vibratory milling experiments, only the most intensified vibratory milling run with the optimal bead size (Run 13) could lead to a nanosuspension comparable (slightly coarser) to that prepared by the wet stirred media milling (Run 14).

\section{CONCLUSIONS}

Process intensification of the vibratory milling through an increase in vibration intensity and bead loading with the optimal bead size can lead to significantly faster production of drug nanosuspensions. The bead selection has been shown to play a major role in the breakage kinetics; optimum bead size decreases with the power density. Microhydrodynamic considerations have offered insight into the roles of process parameters and bead size optimality. PXRD and DSC results do not indicate significant changes to drug crystallinity upon vibratory milling. Compared with the as-received drug particles, vibratory milled particles exhibit markedly fast dissolution, within 2 min, in water due to their large surface area and enhanced wettability imparted by the stabilizers. The vibratory milling has led to slower breakage and coarser nanoparticles than wet stirred media milling, which suggests further process optimization and design modifications, including a jacketed vessel with integrated chiller. With enhanced efficiency, the intensified process may allow the vibratory milling, which is ideally suited as a drug-sparing approach in early development, to prepare a similar nanosuspension to that prepared by wet stirred media milling, which is well-suited to pilot and large-scale production, keeping the milling time the same.

\section{ACKNOWLEDGMENTS}

The authors report financial support through Grant EEC0540855 from NSF ERC for Structured Organic Particulate Systems. Nisso America Inc. is also noted for the kind donation of HPC. The authors would also like to acknowledge Dr. Peter Lucon from Resodyn Acoustic Mixers, Inc. for providing us information about the power calculation and the Excel code.

\section{REFERENCES}

1. Kipp JE. The role of solid nanoparticle technology in the parenteral delivery of poorly water-soluble drugs. Int J Pharm. 2004;284:109-22.

2. Lipinski CA. Poor aqueous solubility: an industry wide problem in drug discovery. Am Pharm Rev. 2002;5:82-5.

3. Adamson A, Gast A. Physical chemical of surfaces. New York: Wiley; 1997.

4. Singh SK, Srinivasan K, Gowthamarajan K, Singare DS, Prakash $\mathrm{D}$, Gaikwad NB. Investigation of preparation parameters of nanosuspension by top-down media milling to improve the dissolution of poorly water-soluble glyburide. Eur J Pharm Biopharm. 2011;78:441-6.

5. Tanaka Y, Inkyo M, Yumoto R, Nagai J, Takano M, Nagata S. Nanoparticulation of probucol, a poorly water-soluble drug, using a novel wet-milling process to improve in vitro dissolution and in vivo oral absorption. Drug Dev Ind Pharm. 2012;38:1015-23.

6. Noyes AA, Whitney WR. The rate of solution of solid substances in their own solutions. J Am Chem Soc. 1897;19:930-4.

7. Basa S, Muniyappan T, Karatgi P, Prabhu R, Pillai R. Production and in vitro characterization of solid dosage form incorporating drug nanoparticles. Drug Dev Ind Pharm. 2008;34:1209-18.

8. Bhakay A, Davé R, Bilgili E. Recovery of BCS class II drugs during aqueous redispersion of core-shell type nanocomposite particles produced via fluidized bed coating. Powder Technol. 2013;236:221-34.

9. Bhakay A, Azad M, Vizzotti E, Dave RN, Bilgili E. Enhanced recovery and dissolution of griseofulvin nanoparticles from surfactant-free nanocomposite microparticles incorporating wetmilled swellable dispersants. Drug Dev Ind Pharm. 2014;40:1509-22.

10. Van Eerdenbrugh B, Van den Mooter G, Augustijns P. Top-down production of drug nanocrystals: nanosuspension stabilization, miniaturization and transformation into solid products. Int $\mathbf{J}$ Pharm. 2008;364:64-75.

11. Sievens-Figueroa L, Bhakay A, Jerez-Rozo JI, Pandya N, Romañach RJ, Michniak-Kohn B, et al. Preparation and characterization of hydroxypropyl methyl cellulose films containing stable BCS class II drug nanoparticles for pharmaceutical applications. Int J Pharm. 2012;423:496-508.

12. Cooper ER. Nanoparticles: a personal experience for formulating poorly water soluble drugs. J Control Release. 2010;141:300-2.

13. Müller RH, Gohla S, Keck CM. State of the art of nanocrystals-special features, production, nanotoxicology aspects and intracellular delivery. Eur J Pharm Biopharm. 2011;78:1-9. 
14. Leleux J, Williams RO. Recent advancements in mechanical reduction methods: particulate systems. Drug Dev Ind Pharm. 2013:1-12.

15. Sun B, Yeo Y. Nanocrystals for the parenteral delivery of poorly water-soluble drugs. Curr Opin Solid St M. 2012;16:295-301.

16. Azad MA, Knieke C, To D, Davé R. Preparation of concentrated stable fenofibrate suspensions via liquid antisolvent precipitation. Drug Dev Ind Pharm. 2014;40:1693-703.

17. Duarte ARC, Costa MS, Simplício AL, Cardoso MM, Duarte CMM. Preparation of controlled release microspheres using supercritical fluid technology for delivery of anti-inflammatory drugs. Int J Pharm. 2006;308:168-74.

18. Chen X, Young TJ, Sarkari M, Williams Iii RO, Johnston KP. Preparation of cyclosporine A nanoparticles by evaporative precipitation into aqueous solution. Int J Pharm. 2002;242:3-14.

19. Shegokar R, Müller RH. Nanocrystals: industrially feasible multifunctional formulation technology for poorly soluble actives. Int J Pharm. 2010;399:129-39.

20. Merisko-Liversidge E, Liversidge GG. Nanosizing for oral and parenteral drug delivery: a perspective on formulating poorlywater soluble compounds using wet media milling technology. Adv Drug Deliver Rev. 2011;63:427-40.

21. Keck CM, Müller RH. Drug nanocrystals of poorly soluble drugs produced by high pressure homogenisation. Eur J Pharm Biopharm. 2006;62:3-16.

22. Juhnke M, Märtin D, John E. Generation of wear during the production of drug nanosuspensions by wet media milling. Eur $\mathrm{J}$ Pharm Biopharm. 2012;81:214-22.

23. Kumar S, Burgess DJ. Wet milling induced physical and chemical instabilities of naproxen nano-crystalline suspensions. Int $\mathbf{J}$ Pharm. 2014;466:223-32.

24. Afolabi A, Akinlabi O, Bilgili E. Impact of process parameters on the breakage kinetics of poorly water-soluble drugs during wet stirred media milling: a microhydrodynamic view. Eur J Pharm Sci. 2014;51:75-86.

25. Kesisoglou F, Panmai S, Wu Y. Nanosizing-oral formulation development and biopharmaceutical evaluation. Adv Drug Deliver Rev. 2007;59:631-44.

26. Merisko-Liversidge EM, Liversidge GG. Drug nanoparticles: formulating poorly water-soluble compounds. Toxicol Pathol. 2008;36:43-8.

27. Monteiro A, Afolabi A, Bilgili E. Continuous production of drug nanoparticle suspensions via wet stirred media milling: a fresh look at the Rehbinder effect. Drug Dev Ind Pharm. 2013;39:266-83.

28. Müller RH, Jacobs C, Kayser O. Nanosuspensions as particulate drug formulations in therapy: rationale for development and what we can expect for the future. Adv Drug Deliver Rev. 2001;47:3-19.

29. Brough C, Williams III RO. Amorphous solid dispersions and nano-crystal technologies for poorly water-soluble drug delivery. Int J Pharm. 2013;453:157-66.

30. Leung DH, Lamberto DJ, Liu L, Kwong E, Nelson T, Rhodes T, et al. A new and improved method for the preparation of drug nanosuspension formulations using acoustic mixing technology. Int J Pharm. 2014;473:10-9.

31. Kardous F, Yahiaoui R, Aoubiza B, Manceau JF. Acoustic mixer using low frequency vibration for biological and chemical applications. Sensor Actuat A-Phys. 2014;211:19-26.

32. Shrivastava A, Sakthivel S, Pitchumani B, Rathore A. A statistical approach for estimation of significant variables in wet attrition milling. Powder Technol. 2011;211:46-53.

33. Connell Jr TL, Risha GA, Yetter RA, Young G, Sundaram DS, Yang V. Combustion of alane and aluminum with water for hydrogen and thermal energy generation. P Combust Inst. 2011;33:1957-65.
34. Sullivan KT, Kuntz JD, Gash AE. The role of fuel particle size on flame propagation velocity in thermites with a nanoscale oxidizer. Propell Explos Pyrot. 2014;39:407-15.

35. Capece M, Huang Z, To D, Aloia M, Muchira C, Davé RN, et al. Prediction of porosity from particle scale interactions: surface modification of fine cohesive powders. Powder Technol. 2014;254:103-13.

36. Huang Z, Scicolone JV, Han X, Davé RN. Improved blend and tablet properties of fine pharmaceutical powders via dry particle coating. Int J Pharm. 2015;478:447-55.

37. Knieke C, Azad MA, To D, Bilgili E, Davé RN. Sub-100 micron fast dissolving nanocomposite drug powders. Powder Technol. 2015;271:49-60.

38. Mullarney MP, Beach LE, Langdon BA, Polizzi MA. Applying dry powder coatings. Pharm Technol. 2011;35:94-102.

39. Howe HW, Warriner JJ, Cook AM, Coguill SL, Farrar LC. Apparatus and method for resonant-vibratory mixing. US Patent, 2007; US7188993 B1.

40. Howe HW, Warriner JJ, Cook AM, Coguill SL, Farrar LC. Method for resonant-vibratory mixing. US Patent, 2011; US7866878 B2.

41. Lim J-Y, Moon J-D. Multiphase mixing apparatus using acoustic resonance. US Patent, 2001; US6283626 B1.

42. Ghosh I, Schenck D, Bose S, Ruegger C. Optimization of formulation and process parameters for the production of nanosuspension by wet media milling technique: effect of vitamin E TPGS and nanocrystal particle size on oral absorption. Eur J Pharm Sci. 2012;47:718-28.

43. Peltonen L, Hirvonen J. Pharmaceutical nanocrystals by nanomilling: critical process parameters, particle fracturing and stabilization methods. J Pharm Pharmacol. 2010;62:1569-79.

44. Li M, Yaragudi N, Afolabi A, Dave R, Bilgili E. Sub-100 nm drug particle suspensions prepared via wet milling with low bead contamination through novel process intensification. Chem Eng Sci. 2015;130:207-20.

45. Bilgili E, Afolabi A. A combined microhydrodynamics-polymer adsorption analysis for elucidation of the roles of stabilizers in wet stirred media milling. Int J Pharm. 2012;439:193-206.

46. Bilgili E, Li M, Afolabi A. Is the combination of cellulosic polymers and anionic surfactants a good strategy for ensuring physical stability of BCS Class II drug nanosuspensions? Pharm Dev Technol. 2015. doi:10.3109/10837450.2015.1022788.

47. Bilgili E, Hamey R, Scarlett B. Production of pigment nanoparticles using a wet stirred mill with polymeric media. China Particuology. 2004;2:93-100.

48. Ghosh I, Bose S, Vippagunta R, Harmon F. Nanosuspension for improving the bioavailability of a poorly soluble drug and screening of stabilizing agents to inhibit crystal growth. Int J Pharm. 2011;409:260-8.

49. Azad M, Afolabi A, Bhakay A, Leonardi J, Davé R, Bilgili E. Enhanced physical stabilization of fenofibrate nanosuspensions via wet co-milling with a superdisintegrant and an adsorbing polymer. Eur J Pharm Biopharm. 2015;94:372-85.

50. Yang H, Teng F, Wang P, Tian B, Lin X, Hu X, et al. Investigation of a nanosuspension stabilized by Soluplus $®$ to improve bioavailability. Int J Pharm. 2014;477:88-95.

51. Patel C, Chakraborty M, Murthy ZVP. Preparation of fenofibrate nanoparticles by combined stirred media milling and ultrasonication method. Ultrason Sonochem. 2014;21:1100-7.

52. Eskin D, Zhupanska O, Hamey R, Moudgil B, Scarlett B. Microhydrodynamic analysis of nanogrinding in stirred media mills. AlChE J. 2005;51:1346-58. 